\title{
Mutations in TGFBR2 gene cause spontaneous cervical artery dissection
}

\author{
Alessandro Pezzini, ${ }_{1}^{1}$ Bruno Drera, ${ }^{2}$ Elisabetta Del Zotto, ${ }^{1}$ Marco Ritelli, ${ }^{2}$ \\ Monica Carletti, ${ }^{3}$ Gianpaolo Tomelleri, ${ }^{3}$ Paolo Bovi, ${ }^{3}$ Alessia Giossi, ${ }^{1}$ Irene Volonghi, ${ }^{1}$ \\ Paolo Costa, ${ }^{1}$ Mauro Magoni, ${ }^{4}$ Alessandro Padovani, ${ }^{1}$ Sergio Barlati, ${ }^{2}$ \\ Marina Colombi ${ }^{2}$
}

\begin{abstract}
- Additional tables are published online only. To view these files please visit the journal online (http://jnnp.bmj.com/).

${ }^{1}$ Dipartimento di Scienze Mediche e Chirurgiche, Clinica Neurologica, Università degli Studi di Brescia, Brescia, Italia ${ }^{2}$ Divisione di Biologia e Genetica, Dipartimento di Scienze Biomediche e Biotecnologie, Università degli Studi di Brescia, Brescia, Italia ${ }^{3}$ UO di Neurologia, Azienda Ospedaliera-Universitaria Borgo Trento, Verona, Italia ${ }^{4}$ Stroke Unit, Neurologia Vascolare, Spedali Civili di Brescia, Brescia, Italia
\end{abstract}

\section{Correspondence to} Dr A Pezzini, Clinica Neurologica, Università degli Studi di Brescia, P le Spedali Civili, 1, 25100 Brescia, Italia; ale_pezzini@hotmail.com

Received 7 October 2010 Revised 9 December 2010 Accepted 15 December 2010 Published Online First 26 January 2011

\begin{abstract}
Mutations in the genes encoding transforming growth factor $\beta$ receptors 1 and 2 (TGFBR1 and TGFBR2) have recently been associated with hereditary connective tissue disorders with widespread vascular involvement, including arterial dissection. To determine whether mutations in these genes cause spontaneous cervical artery dissection (SCAD), all coding exons of TGFBR1 and TGFBR2 were sequenced in 56 consecutive patients with sCAD. Novel TGFBR2 disease causing mutations were found in two patients. The two mutations were the pK327R substitution affecting the kinase domain of TGFBR2 and the $\mathrm{pC} 138 \mathrm{R}$ substitution falling in the extracellular domain of the protein, involved in TGF $\beta$ binding and signalling. No TGFBR1 mutation was found. The findings indicate that TGFBR2 gene mutations are responsible for SCAD in $3.6 \%(95 \% \mathrm{Cl} 0.0$ to 8.4$)$ of cases, have implications in understanding the role of TGF $\beta$ signalling in the pathogenesis of SCAD and emphasise the importance of considering molecular characterisation of the TGFBR2 gene in these patients, regardless of the presence of clinical features suggestive of hereditary connective tissue disorders.
\end{abstract}

Spontaneous cervical artery dissection (sCAD) is a common phenotype, accounting for up to $20 \%$ of ischaemic strokes at a young age. Although its pathogenesis is poorly understood, it is commonly assumed that subtle underlying defects of the connective tissue elements of the extracellular matrix (ECM) might lead to structural instability of the vessel wall and impair its resistance, predisposing to dissection. ${ }^{1}$ Recently, the prominent role of transforming growth factor $\beta$ (TGF $\beta$ ) signalling in the pathogenesis of arterial disease has been established. In particular, mutations of the TGF $\beta$ receptor type 1 and 2 genes (TGFBR 1 and TGFBR2) have been found in patients with overlapping connective tissue disorders-namely, Loeys-Dietz syndrome (LDS), thoracic aneurysm and dissection, Furlong syndrome and Shprintzen-Goldberg syndrome. The phenotypic spectrum of these disorders includes widespread cardiovascular involvement and, especially in LDS, an aggressive vascular disease, with arterial tortuosity and strong predisposition for aneurysms and dissection through the arterial tree, ${ }^{2} 3$ combined with typical craniofacial and skeletal features (LDS type I), or similar to that observed in vascular Ehlers-Danlos syndrome (LDS type II). To date, disease causing mutations in TGFBR genes have been detected in three patients with $\mathrm{sCAD}^{4}$ and no information is available on their prevalence in this vascular disorder.

\section{SUBJECTS AND METHODS}

Patients consecutively admitted to our department between March 2008 and February 2010 were included. The diagnosis of $\mathrm{CAD}$ was confirmed by $\mathrm{MRI} / \mathrm{MR}$ angiography or conventional angiography. The presence of the double lumen sign (a false lumen or an intimal flap), luminal narrowing with the 'string sign' and gradual tapering ending in total occlusion of the lumen (flame-like occlusion) were considered reliable angiographic findings of $\mathrm{CAD}$ whereas a narrowed lumen surrounded by a semilunar shaped intramural haematoma on axial T1 weighted images was considered a pathognomonic MR sign. Dissections occurring as an immediate consequence of a major trauma were labelled 'traumatic' and excluded. ${ }^{5}$ Mutations search was also conducted in 500 control chromosomes of Italian healthy blood donors. Informed consent was obtained in agreement with the Italian bioethic laws.

\section{Phenotype analysis}

Clinically detectable signs and family history of connective tissue abnormalities, including craniofacial, skeletal and cutaneous manifestations, were systematically investigated in each subject. Data were collected by two physicians (BD, EDZ) to ensure a homogeneous evaluation.

\section{Mutation analysis}

The molecular analysis of TGFBR1 (reference sequence NM_004612.2) and TGFBR2 (reference sequence NM_003242.5) genes was performed on genomic DNĀ purified from whole blood using Wizard Genomic DNA purification KIT (Promega, Madison, Wisconsin, USA), according to the manufacturer's instructions. In particular, the exons and intron flanking regions of the TGFBR 1 and TGFBR2 genes were amplified by PCR and sequence analysis was performed in both orientations using the BigDye Terminator Cycle Sequencing Kit on the ABI PRISM 3100 automated sequencer (Applied Biosystems, Foster City, California, USA). ${ }^{6}$

\section{RESULTS}

The study group consisted of 56 subjects (table 1 ).

Connective tissue abnormalities were observed in 18 patients (32.1\%; see online supplementary table 1 
Table 1 Demographics and clinical characteristics of the study group

\begin{tabular}{ll}
\hline Characteristic & \\
\hline Age (mean \pm SD) & $41.4 \pm 7.2$ \\
Body mass index $\left(\mathrm{kg} / \mathrm{m}^{2}\right)$ (mean \pm SD) & $24.3 \pm 3.0$ \\
Sex, female (n (\%)) & $19(33.9)$ \\
Hypertension (n (\%)) & $18(32.1)$ \\
Diabetes mellitus (n (\%)) & $1(1.8)$ \\
Smoking (n (\%)) & $13(23.2)$ \\
Hypercholesterolaemia (n (\%)) & $16(28.6)$ \\
Oral contraceptive (n (\%)) & $6(31.6)$ \\
Migraine (n (\%)) & \\
$\quad$ Any migraine & $33(58.9)$ \\
$\quad$ Migraine without aura & $23(41.0)$ \\
$\quad$ Migraine with aura & $7(12.5)$ \\
Carotid artery dissection (n (\%)) & $30(53.6)$ \\
Vertebral artery dissection (n (\%)) & $16(28.6)$ \\
Multiple vessel dissection (n (\%)) & $10(17.8)$ \\
Cerebral ischaemia (n (\%)) & \\
$\quad$ Infarction & $29(51.8)$ \\
$\quad$ Transient ischaemic attack & $16(28.6)$ \\
Local signs ${ }^{*}(\mathrm{n}(\%))$ & $21(37.5)$ \\
\hline
\end{tabular}

*Headache, neck pain, pulsatile tinnitus, cranial nerves involvment or cervical radiculopathy on the side of the dissection.

available online only). Apart from six known and two novel TGFBR1 polymorphisms, and eight known and one novel TGFBR2 polymorphism (see online supplementary table 2 available online only), we identified TGFBR2 disease causing mutations in two patients (3.6\%, $95 \%$ CI 0.0 to 8.4 ).

\section{Patient No 1}

A 39-year-old man with a personal history of arterial hypertension presented with a right-sided headache and Horner syndrome due to dissection of the right internal carotid artery (ICA) resulting in total vessel occlusion. Oral anticoagulation treatment was initiated, targeting an international normalised ratio of between 2 and 3. Four months later, the patient presented with acute cerebral infarct in the territory of the left middle cerebral artery. MRI angiography showed dissection of the left ICA. Physical examination revealed micrognathia, proptosis, absent lingual frenulum, pectus carinatum, velvety skin and dystrophic scars on both knees. A history of recurrent wrist dislocation was reported. Sequence analysis revealed the novel $\mathrm{c} 1115 \mathrm{~A} \rightarrow \mathrm{G}$ transition in exon 4 of the TGFBR2 gene, leading to the pK372R substitution in the kinase domain of the receptor (figure 1A). Segregation analysis in the proband's family disclosed a TGFBR2 mutation in the unaffected mother and one daughter (figure $1 \mathrm{E}$ ) thus suggesting an incomplete penetrance of the mutation, as in the case of other TGFBRs mutations.

\section{Patient No 2}

A 35-year-old woman was admitted because of Horner syndrome due to dissection of the right ICA. She had a personal history of migraine without aura, hypercholesterolaemia, oral contraceptives use and recurrent subluxation of her knees. A patent foramen ovale was detected on echocardiogram. No involvement of skin, craniofacial or skeletal systems was observed. Family history was also unremarkable. Sequence analysis disclosed the novel $\mathrm{c} 412 \mathrm{~T} \rightarrow \mathrm{C}$ mutation in exon 3 of the
Figure 1 Molecular characterisation of patient No 1 and patient No 2 by genomic DNA sequencing. Sequence chromatogram of patient No $1(A)$, showing the position of the heterozygous $\mathrm{C} 1115 \mathrm{~A} \rightarrow \mathrm{G}$ transition in TGFBR2 exon 4, leading to the novel pK372R missense mutation (substitution of a lysine in the kinase domain of the receptor with an arginine), and patient No 2 (C), showing the position of the heterozygous c412T $\rightarrow$ C transition in TGFBR2 exon 3, leading to the novel $\mathrm{pC} 138 \mathrm{R}$ missense mutation. The lysine at position 372 (B) and the cysteine at position 138 (D) in the extracellular domain of the protein are evolutionary conserved in TGFBR2 orthologues. Multiple sequence alignment was performed using CLUSTALW and PolyPhen. (E) Segregation of the pK372R TGFBR2 mutation in the patient's family pedigree. TGFBR1 and TGFBR2, transforming growth factor $\beta$ receptor type 1 and 2 genes.

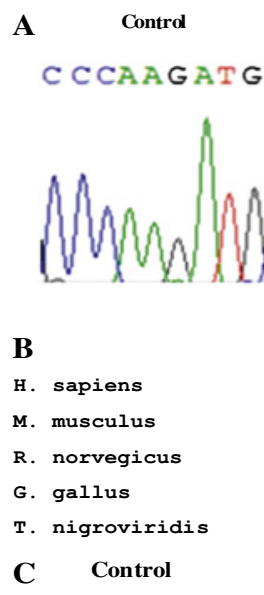

GTT C CTGTA

T P C GR P K M P I VHR

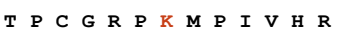

TPCGRPKTPIVHR

T G C G R Y V P V A H

Patient 2

GTT C CNGTA
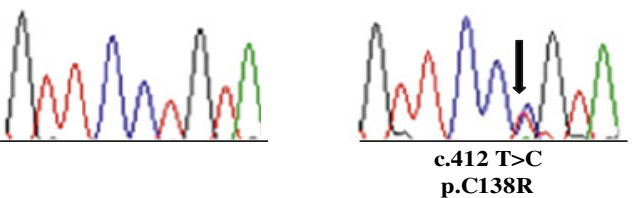

D

H. sapiens

M. musculus

R. norvegicus

G. gallus

T. nigroviridis

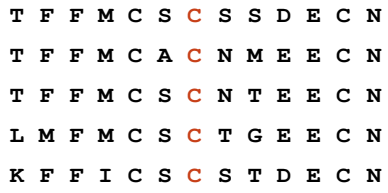

$\mathbf{E}$

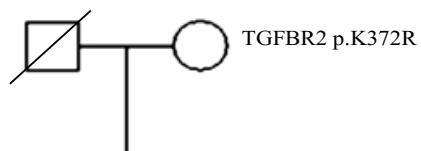


TGFBR2 gene (figure 1C), leading to the substitution of cysteine with arginine in position 138 of the amino acidic sequence (pC138R) in the extracellular domain of the protein. This missense mutation was not detected in the proband's parents, suggesting it arose as a de novo event.

Several arguments support the disease causing effect of the mutations we found: (1) they were not detected in 500 chromosomes of control Italian blood donors; (2) they substitute a highly conserved residue in the orthologues of the receptor (figure $1 \mathrm{~B}, 1 \mathrm{D}$ ); and (3) both mutations are predicted to influence protein function with high probability by PolyPhen tool bioinformatic analysis: the pK372R missense mutation affects a residue in the TGFBR2 kinase domain like the majority of LDS mutations identified to date while the $\mathrm{pC138R}$ missense mutation is the first reported to affect one of the cysteins needed to stabilise the TGFBR2 protein by disulfide bonds between pCys138 and pCys143.

\section{DISCUSSION}

The results of this study indicate that TGFBR2 mutations may be a cause of $\mathrm{sCAD}$, being responsible for $\sim 3.6 \%$ of the cases in the present cohort and, indirectly, prompt speculation on several pathogenic mechanisms of this disease. Firstly, our findings are in line with the hypothesis that TGF $\beta$ is involved in SCAD specific pathways. A number of histological analyses have consistently shown a severe defect in elastogenesis with loss of elastin content and disarrayed elastic fibres in patients with dysregulation of TGF $\beta$ signalling, ${ }^{2} 7$ similar to the ultrastructural abnormalities observed in skin biopsies ${ }^{8}$ and arterial wall specimens ${ }^{9}$ of $\mathrm{sCAD}$ patients. It is conceivable that TGFBR2 mutations may result in altered structure and composition of vascular ECM, predisposing to dissection. Secondly, they reinforce the prevailing idea that $\mathrm{sCAD}$ represents one phenotypic expression of a systemic inherited disorder of the ECM, even in sporadic cases with no other obvious signs of connective tissue abnormalities. In line with this hypothesis, the mutation carriers we identified showed only subtle signs of connective tissue involvement. As in the case of familial thoracic aneurysm and dissection, in which carriers of the defective gene show isolated involvement of the aorta, ${ }^{10}$ we can assume a clinical presentation limited to cervical arteries as part of the phenotypic spectrum of TGFBR2 mutations.

Although mutations in TGFBR2 appear to be an infrequent cause of $\mathrm{sCAD}$, the identification of subjects who carry the defective gene has important clinical implications as regards individual and familial counselling. Because of the aggressive nature of their arteriopathy, mutation carriers require close monitoring of the arterial tree and should be advised of the risk of other life threatening manifestations, such as cervical spine instability and organ rupture. ${ }^{2} 3$ As our findings indicate, TGFBR screening is likely to be recommended only in selected $S C A D$ cases. Unfortunately, defining which $\mathrm{SCAD}$ patients should be screened is not straightforward. Familial clustering of dissection is evident when the disease involves the thoracic aorta while it is a very rare finding in sCAD. ${ }^{11}$ Furthermore, the wide intrafamilial variability of TGFBR2 mutation related phenotypes, the incomplete penetrance of the mutations and the lack of genotype-phenotype correlations ${ }^{3}$ make it difficult to determine what subgroup of sCAD patients is more likely to carry the defective gene and should undergo molecular characterisation. In particular, it is uncertain whether searching for connective tissue abnormalities or specific vascular phenotypes might increase the probability of identifying mutations carriers. As a consequence, awaiting confirmation of our findings in larger series and further data on what specific phenotypes, if any, may be of help in patient selection, it seems advisable to consider the possibility of performing molecular characterisation in all patients with $\mathrm{SCAD}$, regardless of the presence of clinical features suggestive of TGFBR mutation.

Competing interests None.

Patient consent Obtained.

Provenance and peer review Not commissioned; externally peer reviewed.

\section{REFERENCES}

1. Debette S, Leys D. Cervical-artery dissections: predisposing factors, diagnosis, and outcome. Lancet Neurol 2009;8:668-78.

2. Loeys BL, Chen J, Neptune ER, et al. A syndrome of altered cardiovascular, craniofacial, neurocognitive and skeletal development caused by mutations in TGFBR1 or TGFBR2. Nat Genet 2005;37:275-81.

3. Loeys BL, Schwarze U, Holm T, et al. Aneurysm syndromes caused by mutations in the TGF-beta receptor. N Engl J Med 2006;355:788-98.

4. Tran-Fadulu V, Pannu H, Kim DH, et al. Analysis of multigenerational families with thoracic aortic aneurysms and dissections due to TGFBR1 or TGFBR2 mutations. J Med Genet 2009;46:607-13.

5. Pezzini A, Del Zotto E, Archetti S, et al. Plasma homocysteine concentration, C677T MTHFR genotype and 844ins68bp CBS genotype in young adults with spontaneous cervical artery dissection and atherothrombotic stroke. Stroke 2002;33:664-9.

6. Drera B, Tadini G, Barlati S, et al. Identification of a novel TGFBR1 mutation in a Loeys-Dietz syndrome type II patient with vascular Ehlers-Danlos syndrome phenotype. Clin Genet 2008;73:290-3.

7. Singh KK, Rommel K, Mishra A, et al. TGFBR1 and TGFBR2 mutations in patients with features of Marfan syndrome and Loeys-Dietz syndrome. Hum Mutat 2006;27:770-7.

8. Brandt T, Orberk E, Weber R, et al. Pathogenesis of cervical artery dissections. Association with connective tissue abnormalities. Neurology 2001;57:24-30.

9. Völker W, Besselmann M, Dittrich R, et al. Generalized arteriopathy in patients with cervical artery dissection. Neurology 2005;64:1508-13.

10. Pannu H, Tran-Fadulu V, Milewicz DM. Genetic basis of thoracic aortic aneurysms and aortic dissections. Am J Med Genet C Semin Med Genet 2005;139C:10-16

11. Grond-Ginsbach C, Pjontek R, Su Aksay S, et al. Spontaneous arterial dissection: phenotype and molecular pathogenesis. Cell Mol Life Sci 2010;67:1799-815. 


\section{JNNP}

\section{Mutations in TGFBR2 gene cause spontaneous cervical artery dissection}

Alessandro Pezzini, Bruno Drera, Elisabetta Del Zotto, et al.

J Neurol Neurosurg Psychiatry 2011 82: 1372-1374 originally published online January 26,2011

doi: 10.1136/jnnp.2010.231902

Updated information and services can be found at:

http://jnnp.bmj.com/content/82/12/1372.full.html

These include:

Data Supplement

"Web Only Data"

http://jnnp.bmj.com/content/suppl/2011/02/16/jnnp.2010.231902.DC1.html

References This article cites 11 articles, 4 of which can be accessed free at: http://jnnp.bmj.com/content/82/12/1372.full.html\#ref-list-1

Email alerting

Receive free email alerts when new articles cite this article. Sign up in the box at the top right corner of the online article.

Notes

To request permissions go to:

http://group.bmj.com/group/rights-licensing/permissions

To order reprints go to:

http://journals.bmj.com/cgi/reprintform

To subscribe to BMJ go to:

http://group.bmj.com/subscribe/ 